\title{
ANNALES
}

UNIVERSITATIS MARIAE CURIE-SKŁODOWSKA

LUBLIN - POLONIA

MOHAMED K. AOUF and RABHA M. EL-ASHWAH

\section{Differential sandwich theorems for analytic functions defined by Cho-Kwon-Srivastava operator}

\begin{abstract}
By making use of Cho-Kwon-Srivastava operator, we obtain some subordinations and superordinations results for certain normalized analytic functions.
\end{abstract}

1. Introduction. Let $H(U)$ be the class of analytic functions in the open unit disk $U=\{z:|z|<1\}$ and $H(a, n)$ be the subclass of $H(U)$ consisting of functions of the form:

$$
f(z)=a+a_{n} z^{n}+a_{n+1} z^{n+1}+\ldots \quad(a \in C) .
$$

For simplicity, let $H[a]=H[a, 1]$. Also, let $A$ be the subclass of the functions $f \in H(U)$ of the form:

$$
f(z)=z+\sum_{n=2}^{\infty} a_{n} z^{n} .
$$

For $f, g \in H(U)$, we say that the function $f$ is subordinate to $g$, or the function $g$ is superordinate to $f$, if there exists a Schwarz function $w$, i.e., $w \in H(U)$ with $w(0)=0$ and $|w(z)|<1, z \in U$, such that $f(z)=g(w(z))$ for all $z \in U$. This subordination is usually denoted by $f(z) \prec g(z)$. It

2000 Mathematics Subject Classification. 30C45.

Key words and phrases. Hadamard product, Cho-Kwon-Srivastava operator, subordination, superordination. 
is well known that, if the function $g$ is univalent in $U$, then $f(z) \prec g(z)$ is equivalent to $f(0)=g(0)$ and $f(U) \subset g(U)$ (cf., e.g., [7], see also [4]).

Supposing that $p, h$ are two analytic functions in $U$, let

$$
\varphi(r, s, t ; z): C^{3} \times U \rightarrow C .
$$

If $p$ and $\varphi\left(p(z), z p^{\prime}(z), z^{2} p^{\prime \prime}(z) ; z\right)$ are univalent functions in $U$ and if $p$ satisfies the second-order subordination

$$
h(z) \prec \varphi\left(p(z), z p^{\prime}(z), z^{2} p^{\prime \prime}(z) ; z\right),
$$

then $p$ is called to be a solution of the differential superordination (1.2). A function $q \in H(U)$ is called a subordinant of $(1.2)$, if $q(z) \prec p(z)$ for all the functions $p(z)$ satisfying (1.2). A univalent subordinant $\widetilde{q}$ that satisfies $q(z) \prec \widetilde{q}(z)$ for all of the subordinants $q$ of (1.2), is called the best subordinant (cf., e.g., [7], see also [4]).

Recently, Miller and Mocanu [8] obtained sufficient conditions on the functions $h, q$ and $\varphi$ for which the following implication holds:

$$
h(z) \prec \varphi\left(p(z), z p^{\prime}(z), z^{2} p^{\prime \prime}(z) ; z\right) \Rightarrow q(z) \prec p(z) .
$$

For functions $f_{j}(z) \in A$, given by

$$
f_{j}(z)=z+\sum_{n=2}^{\infty} a_{n, j} z^{n} \quad(j=1,2),
$$

we define the Hadamard product (or convolution) of $f_{1}(z)$ and $f_{2}(z)$ by

$$
\left(f_{1} * f_{2}\right)(z)=z+\sum_{n=2}^{\infty} a_{n, 1} a_{n, 2} z^{n}=\left(f_{2} * f_{1}\right)(z) \quad(z \in U) .
$$

In terms of the Pochhammer symbol $(\theta)_{n}$ given by

$$
(\theta)_{n}= \begin{cases}1, & (n=0) \\ \theta(\theta+1) \ldots(\theta+n-1), & (n \in N=\{1,2, \ldots\}),\end{cases}
$$

we now define a function $\varphi(a, c ; z)$ by

$$
\varphi(a, c ; z)=z+\sum_{n=1}^{\infty} \frac{(a)_{n}}{(c)_{n}} z^{n+1}
$$

$\left(a \in R ; c \in R \backslash Z_{0}^{-} ; Z_{0}^{-}=\{0,-1,-2, \ldots\} ; z \in U\right)$.

With the aid of the function $\varphi(a, c ; z)$ defined by (1.3), we consider a function $\varphi^{*}(a, c ; z)$ given by the following convolution

$$
\varphi(a, c ; z) * \varphi^{*}(a, c ; z)=\frac{z}{(1-z)^{\lambda+1}} \quad(\lambda>-1 ; z \in U)
$$

which yields the following family of linear operators $I^{\lambda}(a, c)$ :

$$
I^{\lambda}(a, c) f(z)=\phi^{*}(a, c ; z) * f(z) \quad\left(a, c \in R \backslash Z_{0}^{-} ; \lambda>-1 ; z \in U\right) .
$$


For a function $f(z) \in A$, given by (1.1), it is easily seen from (1.4) that

$$
I^{\lambda}(a, c) f(z)=z+\sum_{n=2}^{\infty} \frac{(c)_{n-1}(\lambda+1)_{n-1}}{(a)_{n-1}(1)_{n-1}} a_{n} z^{n} \quad(z \in U),
$$

which readily yields the following

$$
z\left(I^{\lambda}(a, c) f(z)\right)^{\prime}=(\lambda+1) I^{\lambda+1}(a, c) f(z)-\lambda I^{\lambda}(a, c) f(z)
$$

and

$$
z\left(I^{\lambda}(a+1, c) f(z)\right)^{\prime}=a I^{\lambda}(a, c) f(z)-(a-1) I^{\lambda}(a+1, c) f(z) .
$$

The operator $I^{\lambda}(a, c)$ was introduced and studied by Cho et al. [5].

We also observe that:

(i) $I^{0}(1,1) f(z)=I^{1}(2,1) f(z)=f(z), I^{1}(1,1) f(z)=z f^{\prime}(z)$, $I^{2}(1,1) f(z)=\frac{1}{2}\left(2 z f^{\prime}(z)+z^{2} f^{\prime \prime}(z)\right)$

(ii) $I^{\mu}(\mu+2,1) f(z)=F_{\mu}(f)(z)(\mu>-1)$, where

$$
F_{\mu}(f)(z)=\frac{\mu+1}{z^{\mu}} \int_{0}^{z} t^{\mu-1} f(t) d t \quad(\text { see }[2]) ;
$$

(iii) $I^{0}(n+1,1) f(z)=I_{n} f(z)\left(n \in N_{0}=N \cup\{0\}\right)$ (Noor integral operator, see [11] );

(iv) $I^{\lambda}(\mu+2,1) f(z)=I_{\lambda, \mu} f(z)(\lambda>-1 ; \mu>-2)$ (Choi-Saigo-Srivastava operator see $[6])$.

Recently many authors ([1], [9], [10] and [12]) have used the results of Bulboacă [3] and shown some sufficient conditions applying first order differential subordinations and superordinations.

The main object of the present paper is to find sufficient condition for certain normalized analytic functions $f(z), g(z)$ in $U$ such that $I^{\lambda}(a, c) g(z) \neq 0$ for $0<|z|<1$ and satisfy

$$
q_{1}(z) \prec \frac{I^{\lambda+1}(a, c) f(z)}{I^{\lambda}(a, c) g(z)} \prec q_{2}(z),
$$

where $q_{1}, q_{2}$ are given univalent functions in $U$. Also, we obtain the number of known results as their special cases.

2. Definitions and preliminaries. In order to prove our results, we shall make use of the following known results.

Definition 1 ([8]). Denote by $Q$, the set of all functions $f$ that are analytic and injective on $\bar{U} \backslash E(f)$, where

$$
E(f)=\left\{\zeta \in \partial U: \lim _{z \rightarrow \zeta} f(z)=\infty\right\}
$$

and are such that $f^{\prime}(\zeta) \neq 0$ for $\zeta \in \partial U \backslash E(f)$. 
Lemma 1 ([7]). Let $q$ be univalent in the unit disk $U$ and let $\theta$ and $\varphi$ be analytic in a domain $D$ containing $q(U)$ with $\varphi(w) \neq 0$ when $w \in q(U)$. Set

$$
\psi(z)=z q^{\prime}(z) \varphi(q(z)) \text { and } h(z)=\theta(q(z))+\psi(z) .
$$

Suppose that

(i) $\psi(z)$ is starlike univalent in $U$,

(ii) $\operatorname{Re}\left\{\frac{z h^{\prime}(z)}{\psi(z)}\right\}>0, \quad z \in U$.

If $p$ is analytic in $U$ with $p(0)=q(0), p(U) \subseteq D$ and

$$
\theta(p(z))+z p^{\prime}(z) \varphi(p(z)) \prec \theta(q(z))+z q^{\prime}(z) \varphi(q(z)),
$$

then

$$
p(z) \prec q(z)
$$

and $q$ is the best dominant.

Lemma 2 ([3]). Let $q$ be convex univalent in the unit disk $U$ and let $\theta$ and $\varphi$ be analytic in a domain $D$ containing $q(U)$. Suppose that

(i) $\operatorname{Re}\left\{\frac{\theta^{\prime}(q(z))}{\varphi(q(z))}\right\}>0, \quad z \in U$,

(ii) $\psi(z)=z q^{\prime}(z) \varphi(q(z))$ is starlike univalent in $U$.

If $p \in H[q(0), 1] \cap Q$ with $p(U) \subseteq D$ and $\theta(p(z))+z p^{\prime}(z) \varphi(p(z))$ is univalent in $U$ and

$$
\theta(q(z))+z q^{\prime}(z) \varphi(q(z)) \prec \theta(p(z))+z p^{\prime}(z) \varphi(p(z)),
$$

then

$$
q(z) \prec p(z),
$$

and $q$ is the best subordinant of (2.2).

3. Subordination results. Using Lemma 1, we first prove the following theorem.

Theorem 1. Let $\alpha \neq 0, \beta>0$ and $q(z)$ be convex univalent in $U$ with $q(0)=1$. Further assume that

$$
\operatorname{Re}\left\{\frac{\beta-\alpha}{\alpha}+2 q(z)+\left(1+\frac{z q^{\prime \prime}(z)}{q^{\prime}(z)}\right)\right\}>0 \quad(z \in U) .
$$

If $f, g \in A$ satisfy

$$
\gamma(f, g, \alpha, \beta) \prec(\beta-\alpha) q(z)+\alpha q^{2}(z)+\alpha z q^{\prime}(z),
$$


where

$$
\begin{aligned}
\gamma(f, g, \alpha, \beta)= & (\beta-2 \alpha) \frac{I^{\lambda+1}(a, c) f(z)}{I^{\lambda}(a, c) g(z)}+\alpha\left(\frac{I^{\lambda+1}(a, c) f(z)}{I^{\lambda}(a, c) g(z)}\right)^{2} \\
& +\alpha(\lambda+2) \frac{I^{\lambda+2}(a, c) f(z)}{I^{\lambda}(a, c) g(z)} \\
& -\alpha(\lambda+1) \frac{I^{\lambda+1}(a, c) g(z)}{I^{\lambda}(a, c) g(z)}\left(\frac{I^{\lambda+1}(a, c) f(z)}{I^{\lambda}(a, c) g(z)}\right)
\end{aligned}
$$

then

$$
\frac{I^{\lambda+1}(a, c) f(z)}{I^{\lambda}(a, c) g(z)} \prec q(z)
$$

and $q$ is the best dominant.

Proof. Define the function $p(z)$ by

$$
p(z)=\frac{I^{\lambda+1}(a, c) f(z)}{I^{\lambda}(a, c) g(z)} \quad(z \in U) .
$$

Then the function $p(z)$ is analytic in $U$ and $p(0)=1$. Therefore, differentiating (3.4) logarithmically with respect to $z$ and using the identity (1.6) in the resulting equation, we have

$$
\begin{aligned}
& \frac{I^{\lambda+1}(a, c) f(z)}{I^{\lambda}(a, c) g(z)}\left[\beta-2 \alpha+\alpha \frac{I^{\lambda+1}(a, c) f(z)}{I^{\lambda}(a, c) g(z)}\right. \\
& \left.+\alpha(\lambda+2) \frac{I^{\lambda+2}(a, c) f(z)}{I^{\lambda+1}(a, c) f(z)}-\alpha(\lambda+1) \frac{I^{\lambda+1}(a, c) g(z)}{I^{\lambda}(a, c) g(z)}\right] \\
& =(\beta-\alpha) p(z)+\alpha p^{2}(z)+\alpha z p^{\prime}(z) .
\end{aligned}
$$

By using (3.5) in (3.2), we have

$$
(\beta-\alpha) p(z)+\alpha p^{2}(z)+\alpha z p^{\prime}(z) \prec(\beta-\alpha) q(z)+\alpha q^{2}(z)+\alpha z q^{\prime}(z) .
$$

By setting

$$
\theta(w)=\alpha w^{2}+(\beta-\alpha) w \quad \text { and } \quad \varphi(w)=\alpha,
$$

we can easily observe that $\theta(w)$ and $\varphi(w)$ are analytic in $C \backslash\{0\}$ and that $\varphi(w) \neq 0$. Hence the result now follows by using Lemma 1 .

Remark 1. Putting $\lambda=0, a=c=1$ and taking $f(z) \equiv g(z)(z \in U)$ in Theorem 1, we obtain the result obtained by Murugusundaramoorthy and Magesh [10, Corollary 2.9].

Putting $f(z) \equiv g(z)(z \in U)$ in Theorem 1, we obtain the following corollary. 
Corollary 1. Let $\alpha \neq 0, \beta>0$ and $q$ be convex univalent in $U$ with $q(0)=1$ and (3.1) holds true. If $f \in A$ satisfies

$$
\begin{gathered}
(\beta-2 \alpha) \frac{I^{\lambda+1}(a, c) f(z)}{I^{\lambda}(a, c) f(z)}+\alpha(\lambda+2) \frac{I^{\lambda+2}(a, c) f(z)}{I^{\lambda}(a, c) f(z)}-\alpha \lambda\left(\frac{I^{\lambda+1}(a, c) f(z)}{I^{\lambda}(a, c) f(z)}\right)^{2} \\
\prec(\beta-\alpha) q(z)+\alpha q^{2}(z)+\alpha z q^{\prime}(z),
\end{gathered}
$$

then

$$
\frac{I^{\lambda+1}(a, c) f(z)}{I^{\lambda}(a, c) f(z)} \prec q(z)
$$

and $q$ is the best dominant.

Putting $a=\mu+2(\mu>-2)$ and $c=1$ in Theorem 1, we obtain the following corollary.

Corollary 2. Let $\alpha \neq 0, \beta>0$ and $q$ be convex univalent in $U$ with $q(0)=1$ and (3.1) holds true. If $f, g \in A$ satisfy

$$
\gamma_{1}(f, g, \alpha, \beta) \prec(\beta-\alpha) q(z)+\alpha q^{2}(z)+\alpha z q^{\prime}(z),
$$

where

$$
\begin{aligned}
& \gamma_{1}(f, g, \alpha, \beta)=(\beta-2 \alpha) \frac{I_{\lambda+1, \mu} f(z)}{I_{\lambda, \mu} g(z)}+\alpha\left(\frac{I_{\lambda+1, \mu} f(z)}{I_{\lambda, \mu} g(z)}\right)^{2} \\
& \quad+\alpha(\lambda+2) \frac{I_{\lambda+2, \mu} f(z)}{I_{\lambda, \mu} g(z)}-\alpha(\lambda+1) \frac{I_{\lambda+1, \mu} g(z)}{I_{\lambda, \mu} g(z)}\left(\frac{I_{\lambda+1, \mu} f(z)}{I_{\lambda, \mu} g(z)}\right),
\end{aligned}
$$

then

and $q$ is the best dominant.

$$
\frac{I_{\lambda+1, \mu} f(z)}{I_{\lambda, \mu} g(z)} \prec q(z)
$$

Putting $a=\mu+2(\mu>-1), c=1$ and $\lambda=\mu$ in Theorem 1, we obtain the following corollary.

Corollary 3. Let $\alpha \neq 0, \beta>0$ and $q$ be convex univalent in $U$ with $q(0)=1$ and (3.1) holds true. If $f, g \in A$ satisfy

$$
\gamma_{2}(f, g, \alpha, \beta) \prec(\beta-\alpha) q(z)+\alpha q^{2}(z)+\alpha z q^{\prime}(z),
$$

where

$$
\begin{aligned}
\gamma_{2}(f, g, \alpha, \beta)= & (\beta-\alpha+\alpha \mu) \frac{f(z)}{F_{\mu}(g)(z)}+\alpha\left(\frac{f(z)}{F_{\mu}(g)(z)}\right)^{2} \\
& +\alpha \frac{z f^{\prime}(z)}{F_{\mu}(g)(z)}-\alpha(\mu+1) \frac{g(z)}{F_{\mu}(g)(z)} \frac{f(z)}{F_{\mu}(g)(z)},
\end{aligned}
$$

then

$$
\frac{f(z)}{F_{\mu}(g)(z)} \prec q(z)
$$

and $q$ is the best dominant. 
Putting $f(z) \equiv g(z)(z \in U)$ in Corollary 3, we obtain the following corollary.

Corollary 4. Let $\alpha \neq 0, \beta>0$ and $q$ be convex univalent in $U$ with $q(0)=1$ and (3.1) holds true. If $f \in A$ satisfies

$$
\gamma_{3}(f, \alpha, \beta) \prec(\beta-\alpha) q(z)+\alpha q^{2}(z)+\alpha z q^{\prime}(z),
$$

where

$$
\gamma_{3}(f, \alpha, \beta)=(\beta-\alpha+\alpha \mu) \frac{f(z)}{F_{\mu}(f)(z)}+\alpha \frac{z f^{\prime}(z)}{F_{\mu}(f)(z)}-\alpha \mu\left(\frac{f(z)}{F_{\mu}(f)(z)}\right)^{2}
$$

then

$$
\frac{f(z)}{F_{\mu}(f)(z)} \prec q(z) \quad(\mu>-1),
$$

and $q$ is the best dominant.

\section{Superordination and sandwich results.}

Theorem 2. Let $\alpha \neq 0$ and $\beta>0$. Let $q$ be convex univalent in $U$ with $q(0)=1$. Assume that

$$
\operatorname{Re}\{q(z)\} \geq \operatorname{Re}\left\{\frac{\alpha-\beta}{2 \alpha}\right\} .
$$

Let $f, g \in A, \frac{I^{\lambda+1}(a, c) f(z)}{I^{\lambda}(a, c) g(z)} \in H[q(0), 1] \cap Q$. Let $\gamma(f, g, \alpha, \beta)$ be univalent in $U$ and

$$
(\beta-\alpha) q(z)+\alpha q^{2}(z)+\alpha z q^{\prime}(z) \prec \gamma(f, g, \alpha, \beta),
$$

where $\gamma(f, g, \alpha, \beta)$ is given by (3.3), then

$$
q(z) \prec \frac{I^{\lambda+1}(a, c) f(z)}{I^{\lambda}(a, c) g(z)}
$$

and $q$ is the best subordinant.

Proof. Let $p(z)$ be defined by (3.4). Therefore, differentiating (3.4) with respect to $z$ and using the identity (1.6) in the resulting equation, we have

$$
\gamma(f, g, \alpha, \beta)=(\beta-\alpha) p(z)+\alpha p^{2}(z)+\alpha z p^{\prime}(z),
$$

then

$$
(\beta-\alpha) q(z)+a q^{2}(z)+\alpha z q^{\prime}(z) \prec(\beta-\alpha) p(z)+\alpha p^{2}(z)+\alpha z p^{\prime}(z) .
$$

By setting $\theta(w)=\alpha w^{2}+(\beta-\alpha) w$ and $\varphi(w)=\alpha$, it is easily observed that $\theta(w)$ is analytic in $C$. Also, $\varphi(w)$ is analytic in $C \backslash\{0\}$ and that $\varphi(w) \neq 0$. Since $q(z)$ is convex univalent, it follows that

$$
\operatorname{Re}\left\{\frac{\theta^{\prime}(q(z))}{\varphi(q(z))}\right\}=\operatorname{Re}\left\{\frac{\beta-\alpha}{\alpha}+2 q(z)\right\}>0 \quad(z \in U) .
$$


Now Theorem 2 follows by applying Lemma 2 .

Putting $f(z) \equiv g(z)(z \in U)$ in Theorem 2, we obtain the following corollary.

Corollary 5. Let $\alpha \neq 0, \beta \geq 1$ and $q$ be convex univalent in $U$ with $q(0)=1$ and (4.1) holds true. Let $f \in A, \frac{I^{\lambda+1}(a, c) f(z)}{I^{\lambda}(a, c) f(z)} \in H[q(0), 1] \cap Q$. Let

$$
\begin{aligned}
\gamma(f, \alpha, \beta)= & (\beta-2 \alpha) \frac{I^{\lambda+1}(a, c) f(z)}{I^{\lambda}(a, c) f(z)}+\alpha(\lambda+2) \frac{I^{\lambda+2}(a, c) f(z)}{I^{\lambda}(a, c) f(z)} \\
& -\alpha \lambda\left(\frac{I^{\lambda+1}(a, c) f(z)}{I^{\lambda}(a, c) f(z)}\right)^{2},
\end{aligned}
$$

be univalent in $U$ and

$$
(\beta-\alpha) q(z)+a q^{2}(z)+\alpha z q^{\prime}(z) \prec \gamma(f, \alpha, \beta),
$$

then

$$
q(z) \prec \frac{I^{\lambda+1}(a, c) f(z)}{I^{\lambda}(a, c) f(z)},
$$

and $q$ is the best subordinant.

Putting $a=\mu+2(\mu>-2)$ and $c=1$ in Theorem 2, we obtain the following corollary.

Corollary 6. Let $\alpha \neq 0, \beta \geq 1$ and $q$ be convex univalent in $U$ with $q(0)=1$ and (4.1) holds true. Let $f, g \in A, \frac{I_{\lambda+1, \mu} f(z)}{I_{\lambda, \mu} g(z)} \in H[q(0), 1] \cap Q$. Let $\gamma_{1}(f, g, \alpha, \beta)$ be univalent in $U$ and

$$
(\beta-\alpha) q(z)+\alpha q^{2}(z)+\alpha z q^{\prime}(z) \prec \gamma_{1}(f, g, \alpha, \beta),
$$

where $\gamma_{1}(f, g, \alpha, \beta)$ is given by (3.7), then

$$
q(z) \prec \frac{I_{\lambda+1, \mu} f(z)}{I_{\lambda, \mu} g(z)},
$$

and $q$ is the best subordinant.

Putting $a=\mu+2(\mu>-1), c=1$ and $\lambda=\mu$ in Theorem 2, we obtain the following corollary.

Corollary 7. Let $\alpha \neq 0, \beta \geq 1$ and $q$ be convex univalent in $U$ with $q(0)=1$ and (4.1) holds true. Let $f, g \in A, \frac{f(z)}{F_{\mu}(g)(z)} \in H[q(0), 1] \cap Q$. Let $\gamma_{2}(f, g, \alpha, \beta)$ be univalent in $U$ and

$$
(\beta-\alpha) q(z)+\alpha q^{2}(z)+\alpha z q^{\prime}(z) \prec \gamma_{2}(f, g, \alpha, \beta),
$$


where $\gamma_{2}(f, g, \alpha, \beta)$ is given by (3.8), then

$$
q(z) \prec \frac{f(z)}{F_{\mu}(g)(z)},
$$

and $q$ is the best subordinant.

Putting $f(z) \equiv g(z)(z \in U)$ in Corollary 7 , we obtain the following corollary.

Corollary 8. Let $\alpha \neq 0, \beta \geq 1$ and $q$ be convex univalent in $U$ with $q(0)=1$ and (4.1) holds true. Let $f \in A, \frac{f(z)}{F_{\mu}(f)(z)} \in H[q(0), 1] \cap Q$. Let $\gamma_{3}(f, \alpha, \beta)$ be univalent in $U$ and

$$
(\beta-\alpha) q(z)+\alpha q^{2}(z)+\alpha z q^{\prime}(z) \prec \gamma_{3}(f, \alpha, \beta),
$$

where $\gamma_{3}(f, \alpha, \beta)$ is given by (3.9), then

$$
q(z) \prec \frac{f(z)}{F_{\mu}(f)(z)} \quad(\mu>-1),
$$

and $q$ is the best subordinant.

We conclude this section by stating the following sandwich result.

Theorem 3. Let $q_{1}$ and $q_{2}$ be convex univalent in $U, \alpha \neq 0$ and $\beta \geq 1$. Suppose $q_{2}$ satisfies (3.1) and $q_{1}$ satisfies (4.1). Moreover, suppose

$$
\frac{I^{\lambda+1}(a, c) f(z)}{I^{\lambda}(a, c) g(z)} \in H[1,1] \cap Q
$$

and $\gamma(f, g, \alpha, \beta)$ is univalent in $U$. If $f, g \in A$ satisfy

$$
\begin{aligned}
(\beta-\alpha) q_{1}(z)+\alpha q_{1}^{2}(z)+a z q_{1}^{\prime}(z) & \prec \gamma(f, g, \alpha, \beta) \\
& \prec(\beta-\alpha) q_{2}(z)+\alpha q_{2}^{2}(z)+a z q_{2}^{\prime}(z),
\end{aligned}
$$

where $\gamma(f, g, \alpha, \beta)$ is given by (3.3), then

$$
q_{1}(z) \prec \frac{I^{\lambda+1}(a, c) f(z)}{I^{\lambda}(a, c) g(z)} \prec q_{2}(z)
$$

and $q_{1}, q_{2}$ are, respectively, the best subordinant and the best dominant.

By making use of Corollaries 2 and 6, we obtain the following corollary.

Corollary 9. Let $q_{1}$ and $q_{2}$ be convex univalent in $U, \alpha \neq 0$ and $\beta \geq 1$. Suppose $q_{2}$ satisfies (3.1) and $q_{1}$ satisfies (4.1). Moreover, suppose

$$
\frac{I_{\lambda+1, \mu}(a, c) f(z)}{I_{\lambda, \mu}(a, c) g(z)} \in H[1,1] \cap Q
$$


and $\gamma_{1}(f, g, \alpha, \beta)$ is univalent in $U$. If $f, g \in A$ satisfy

$$
\begin{aligned}
(\beta-\alpha) q_{1}(z)+\alpha q_{1}^{2}(z)+\alpha z q_{1}^{\prime}(z) & \prec \gamma_{1}(f, g, \alpha, \beta) \\
& \prec(\beta-\alpha) q_{2}(z)+\alpha q_{2}^{2}(z)+\alpha z q_{2}^{\prime}(z),
\end{aligned}
$$

where $\gamma_{1}(f, g, \alpha, \beta)$ is given by (3.7), then

$$
q_{1}(z) \prec \frac{I_{\lambda+1, \mu} f(z)}{I_{\lambda, \mu} g(z)} \prec q_{2}(z) \quad(\mu>-2)
$$

and $q_{1}, q_{2}$ are, respectively, the best subordinant and the best dominant.

By making use of Corollaries 3 and 7 , we obtain the following corollary.

Corollary 10. Let $q_{1}$ and $q_{2}$ be convex univalent in $U, \alpha \neq 0$ and $\beta \geq 1$. Suppose $q_{2}$ satisfies (3.1) and $q_{1}$ satisfies (4.1). Moreover, suppose

$$
\frac{f(z)}{F_{\mu}(g)(z)} \in H[1,1] \cap Q
$$

and $\gamma_{2}(f, g, \alpha, \beta)$ is univalent in $U$. If $f, g \in A$ satisfy

$$
\begin{aligned}
(\beta-\alpha) q_{1}(z)+\alpha q_{1}^{2}(z)+\alpha z q_{1}^{\prime}(z) & \prec \gamma_{2}(f, g, \alpha, \beta) \\
& \prec(\beta-\alpha) q_{2}(z)+\alpha q_{2}^{2}(z)+\alpha z q_{2}^{\prime}(z),
\end{aligned}
$$

where $\gamma_{2}(f, g, \alpha, \beta)$ is given by (3.8), then

$$
q_{1}(z) \prec \frac{f(z)}{F_{\mu}(g)(z)} \prec q_{2}(z) \quad(\mu>-1)
$$

and $q_{1}, q_{2}$ are, respectively, the best subordinant and the best dominant.

By making use of Corollaries 4 and 8, we obtain the following corollary.

Corollary 11. Let $q_{1}$ and $q_{2}$ be convex univalent in $U, \alpha \neq 0$ and $\beta \geq 1$. Suppose $q_{2}$ satisfies (3.1) and $q_{1}$ satisfies (4.1). Moreover, suppose

$$
\frac{f(z)}{F_{\mu}(f)(z)} \in H[1,1] \cap Q
$$

and $\gamma_{3}(f, \alpha, \beta)$ is univalent in $U$. If $f \in A$ satisfies

$$
\begin{aligned}
(\beta-\alpha) q_{1}(z)+\alpha q_{1}^{2}(z)+\alpha z q_{1}^{\prime}(z) & \prec \gamma_{3}(f, \alpha, \beta) \\
& \prec(\beta-\alpha) q_{2}(z)+\alpha q_{2}^{2}(z)+\alpha z q_{2}^{\prime}(z),
\end{aligned}
$$

where $\gamma_{3}(f, \alpha, \beta)$ is given by (3.9), then

$$
q_{1}(z) \prec \frac{f(z)}{F_{\mu}(f)(z)} \prec q_{2}(z) \quad(\mu>-1)
$$

and $q_{1}, q_{2}$ are, respectively, the best subordinant and the best dominant.

Acknowledgments. The authors thank the referees for their valuable suggestions to improve the paper. 


\section{REFERENCES}

[1] Ali, R. M., Ravichandran, V., Hussain Khan, M. and Subramanian, K. G., Differential sandwich theorems for certain analytic functions, Far East J. Math. Sci. 15, no. 1 (2005), 87-94.

[2] Bernardi, S. D., Convex and starlike univalent functions, Trans. Amer. Math. Soc. 135 (1969), 429-446.

[3] Bulboacă, T., A class of first-order differential superordination, Demonstratio Math. 35, no. 2 (2002), 287-292.

[4] Bulboacă, T., Differential Subordinations and Superordinations, Recent Results, House of Scientific Book Publ., Cluj-Napoca, 2005.

[5] Cho, N. E., Kwon, O. S. and Srivastava, H. M., Inclusion relationships and argument properties for certain subclasses of multivalent functions associated with a family of linear operators, J. Math. Anal. Appl. 292 (2004), 470-483.

[6] Choi, J. H., Saigo, M. and Srivastava, H. M., Some inclusion properties of a certain family of integral operators, J. Math. Anal. Appl. 276 (2002), 432-445.

[7] Miller, S. S., Mocanu, P. T., Differential Subordination Theory and Applications, Marcel Dekker, New York, 2000.

[8] Miller, S. S., Mocanu, P. T., Subordinant of differential superordinations, Complex Var. Theory Appl. 48, no. 10 (2003), 815-826.

[9] Murugusundaramoorthy, G., Magesh, N., Differential subordinations and superordinations for analytic functions defined by Dziok-Srivastava linear operator, JIPAM. J. Inequal. Pure Appl. Math. 7, no. 4 (2006), Art. 152, 9 pp.

[10] Murugusundaramoorthy, G., Magesh, N., Differential sandwich theorem for analytic functions defined by Hadamard product, Ann. Univ. Mariae Curie-Skłodowska Sect. A 61 (2007), 117-127.

[11] Noor, K. I., Noor, M. A., On integral operators, J. Math. Anal. Appl. 238 (1999), 341-352.

[12] Shanmugam, T. N., Ravichandran, V. and Sivasubramanian, S., Differential sandwich theorems for same subclasses of analytic functions, Aust. J. Math. Anal. Appl. 3, no. 1 (2006), Art. 8, 11 pp.

\section{K. Aouf}

Department of Mathematics

Faculty of Science

Mansoura University

Mansoura 35516

Egypt

e-mail: mkaouf127@yahoo.com

Received April 25, 2009

\section{R. M. El-Ashwah}

Department of Mathematics

Faculty of Science

Mansoura University

Mansoura 35516

Egypt

e-mail: $r_{-}$elashwah@yahoo.com 